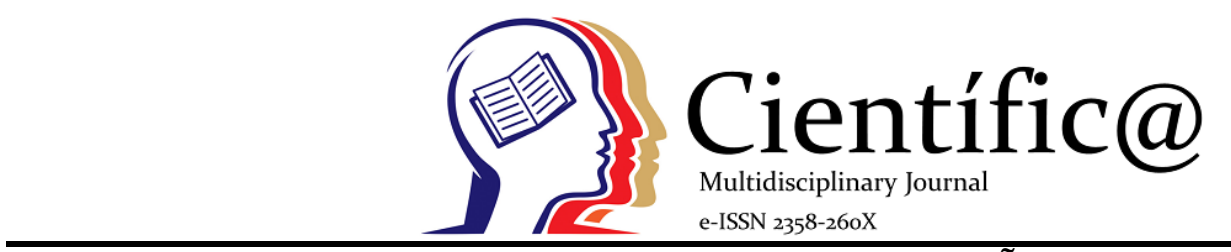

\title{
QUALIDADE DE VIDA DE IDOSOS EM INSTITUIÇÕES DE LONGA PERMANÊNCIA: REVISÃO INTEGRATIVA
}

\author{
QUALITY OF LIFE OF THE ELDERLY IN LONG-TERM INSTITUTIONS: INTEGRATIVE REVIEW
}

\author{
Edna Lopes Santos ${ }^{1}$, Yhasmim Castelo Da Silva ${ }^{1}$, Máriam Hanna Daccache ${ }^{2}$
}

${ }^{1}$ Acadêmico, Universidade Evangélica de Goiás - UniEVANGÉLICA, Departamento de Psicologia.

${ }^{2}$ Professora mestra, Universidade Evangélica de Goiás - UniEVANGÉLICA, Departamento de Psicologia;

\section{Info}

Recebido: 05/2021

Publicado: 06/2021

DOI: $10.37951 / 2358-260 X .2021 v 8 i 1.5862$

ISSN: 2358-260X

\section{Palavras-Chave}

envelhecimento, idoso, instituição de longa permanência, qualidade de vida Keywords:

aging, elderly, long-term institution, quality of life

\section{Resumo}

0 aumento da população idosa, somado às mudanças na constituição familiar e ao fortalecimento da mulher no mercado de trabalho, provocaram o desaparecimento da figura do cuidador. Facetas dessas mudanças resultou na elevação da demanda da institucionalização do idoso. Porém a qualidade prestada por instituições de longa permanência é algo que requer análise, pois pode comprometer a qualidade de vida dos idosos. Sendo assim, este trabalho tem por objetivo analisar a qualidade de vida de idosos em Instituições de Longa Permanência no Brasil. Trata-se de uma revisão integrativa da literatura, realizada nos bancos de dados das plataformas Biblioteca Virtual da Saúde e Scientific Eletronic Library Online de Saúde Pública, com trabalhos publicados no Brasil, entre 2014 e 2019. Após a revisão de 6 artigos eleitos para análise, constatou-se que a qualidade de vida de idosos asilados foi inferior comparado a grupos de idosos não institucionalizados, exceto para idosos em instituições de alto padrão econômico.

\section{Abstract}

The increase in the elderly population, added to the changes in the family constitution and the strengthening of women in the labor market, led to the disappearance of the caregiver figure. Facets of these changes resulted in increased demand for institutionalization of the elderly. However, the quality provided by long-term care institutions is something that requires analysis, as it may compromise the quality of life of the elderly. Thus, this paper aims to analyze the quality of life of the elderly in long-term care institutions in Brazil. This is an integrative literature review, performed in the databases of the Virtual Health Library and Scientific Electronic Library Online Public Health platforms, with works published in Brazil, between 2014 and 2019. After reviewing 6 articles elected for analysis, it was found that the quality of life of elderly asylum was lower compared to groups of noninstitutionalized elderly, except for elderly in institutions of high economic standard.

\section{INTRODUÇÃO}

De acordo com estudos das Nações Unidas (2011), a população idosa (idade superior a60 anos) está projetada para crescer o triplo até 2050, aproximando-se de dois bilhões de idososem todo o mundo. No Brasil, o Instituto Brasileiro de Geografia e Estatística (IBGE) constatouque, conforme o censo demográfico de 2010 , quase $11 \%$ da população são de idosos, com maior predomínio de mulheres que representam 55,5\% desse total (IBGE, 2010). Segundo os indicadores demográficos atualizados no ano de 2017 pelo IBGE, a expectativa de vida dos brasileiros subiu: para as mulheres chegou a 79,6 anos e para os homens 72,5 anos. Isto indicaum aumento da população idosa e, com isso, surgem diversas preocupações inclusive aquelas relacionadas às políticas públicas que objetivam trabalhar as vulnerabilidades e manter a qualidade de vida dessa população.

A partir da crescente ascensão da população idosa houve um aumento do interesse de 
pesquisadores e profissionais da área da saúde em analisar e estudar métodos de atendimento em gerontologia (Matos, 2019).

Envelhecer é algo dinâmico, contínuo e acarreta diversas mudanças permanentes (Oliveira, 2018). Neste processo de envelhecimento, o indivíduo apresenta alterações psicológicas, sociais e morfofisiológicas, em conjunto com a prevalência de doenças crônicas degenerativas (Campolina, 2013).

A ampliação da expectativa de vida citada acima, tem-se dado em meio a mudanças sociais e de estruturas familiares. Uma das consequências destas mudanças, é o crescente número de idosos que moram sozinhos e não podem usufruir da companhia de pessoas mais jovens ou de um cuidador, ou vivem em instituições de longa permanência (Schiozer, 2011).

No Brasil, o Estatuto do Idoso, garante a permanência de idosos em entidades de longa permanência em casos de inexistência de grupo familiar, casa-lar ou em situações de abandonoou de carência de recursos financeiros (Freire, 2018).

Apesar da importância das Instituições de Longa Permanência para Idosos (ILPI) em situações de abandono, esses locais podem representar situações de isolamento, inatividade física e mental, privação de vida social, afetiva e sexual, o que pode gerar comprometimento na qualidade de vida (Pavan, Meneguel \& Junges, 2008).

A Organização Mundial da Saúde (OMS), define qualidade de vida como "a percepção individual de sua posição na vida dentro do contexto cultural e do sistema de valores em que vive e com respeito aos seus objetivos, expectativas, normas e preocupações.” É considerado um conceito complexo por analisar saúde, estado físico, psicológico, nível de independência, relações sociais, crenças pessoais e a relação com as características relacionadas ao ambiente. (OMS, 1998, p. 9).

\section{Saúde e Idoso}

$\mathrm{Na}$ Constituição Brasileira de 1988, seção II, os artigos 196, 197, 198 e 199 abordaramo conceito de saúde na perspectiva política, econômica e social. O artigo 196 prevê que:

A saúde é um direito de todos e dever do estado, garantido mediante medidas políticas,sociais e econômicas que visem à redução do risco de doença e de agravos e ao acesso universal e igualitário às ações e serviços para a sua promoção, proteção e recuperação(BRASIL, 1998).

Apesar da clássica definição de saúde da OMS, o conceito de saúde pode relacionar-se com diversos fatores, entre eles a conjuntura socioeconômica, política e cultural que o indivíduo está inserido. Desta maneira, entende-se que a concepção de se ter ou não saúde varia de indivíduo para indivíduo. Neste contexto, ao analisar a perspectiva do idoso, envelhecer com saúde pode relacionar-se a diversos fatores biopsicossociais e ambientais (Bimler \& Uusküla, 2018).

De acordo com a Lei $n^{\circ} 10.741$ de $1^{\circ}$ de outubro de 2003, que institui o Estatuto do Idosono Brasil, considera-se uma pessoa idosa quando se tem 60 anos ou mais. Especialistas de estudos sobre o envelhecimento subdivide em três, os grupos de pessoas mais velhas: os idososjovens, refere-se a pessoas de 65 a 74 anos, que costumam estar ativas, cheias de vida; os idososvelhos, de 75 a 84 anos e os idosos mais velhos, de 85 anos ou mais, aqueles que 
têm maior tendência para a fraqueza e para a enfermidade (Papalia, Olds \& Feldman, 2006, citado por Schneider \& Irigaray, 2008).

As pessoas nessa faixa etária necessitam de atendimento multiprofissional para uma melhor compreensão dos aspectos que englobam o processo fisiológico do envelhecimento e suas consequências sociais e psicológicas. Dentre as principais alterações fisiológicas, podem-se citar as limitações de movimento, a sarcopenia e a incontinência urinária, que geram consequências psicológicas e sociais. O idoso, diversas vezes, prefere o isolamento social frente ao medo de urinar involuntariamente em locais públicos ou o constrangimento pela lentificação do movimento, ocasionando sentimento de baixa autoestima e piora na qualidade de vida (QV)(Matos, 2019).

Outro fator relacionado as alterações fisiológicas é a diminuição da capacidade funcional, caracterizada como a capacidade de realizar atividades do cotidiano sem auxílio, quepodem ser de dois tipos: atividade da vida diária (AVD) e atividade instrumental da vida diária(AIVD) (Freitas \& Scheicher, 2010). As AVDs dizem respeito a capacidade de levantar-se de uma cadeira ou cama, deambular e autocuidado para banho, alimentação e vestir-se, enquanto as AIVDs estão relacionadas a socialização, cuidar do próprio dinheiro e realizar compras (Freitas \& Scheicher, 2010). As condições de saúde dos idosos residentes em ILPI variam entre a dependência total, dependência parcial e independência para a realização de atividadesde vida diária (Lima, Gomes, Frota \& Pereira, 2016).

Em relação aos aspectos psicológicos do envelhecimento, a teoria do desenvolvimento ao longo da vida, que é embasada nas pesquisas em psicologia do envelhecimento, também conhecida como lifespan, é considerada um marco teórico para o envelhecer saudável. Essa teoria mudou o ideal de que o idoso é um ser doente e passivo na sociedade, e demonstra que é possível envelhecer e se desenvolver durante todo o curso da vida (Baltes, Lindenberger \& Stadinger, 2006).

Neste sentido, a psicologia contribui para o desenvolvimento saudável no envelhecer por meio de intervenções que podem equilibrar as limitações e pontencialidades do idoso, sendoa aquisão de novas aprendizagens umas das principais atividades que auxilia no envelhecimento saudável (ScoralickLempke \& Barbosa, 2012).

\section{Qualidade de Vida}

De acordo com Celich (2008), a qualidade de vida está relacionada com percepções acerca do bem-estar, da autonomia, da independência, da satisfação pessoal, da produtividade,da continuação dos papéis familiares e estão ligados ao dia a dia de cada sujeito. Estas percepções se relacionam com as capacidades ou habilidades desempenhadas pelo mesmo ao longo do dia, para assim obter satisfação. Para este autor, envelhecer com qualidade de vida consiste na otimização da expectativa de vida, logo, as pessoas poderão viver saudáveis por mais tempo, dependendo dos fatores econômicos e sociais.

Torres, Reis e Fernandes (2009), definem qualidade de vida envolvendo dimensões como: habilidade funcional, função social, bem-estar físico, familiar e emocional, espiritualidade, função ocupacional e sexualidade, que quando agregados sustenta o sujeito emequilíbrio consigo mesmo e com os outros que o cercam.

A qualidade de vida, quando não conduzida de forma preventiva, é comprometida pelas alterações fisiológicas, psíquicas e socioeconômicas. Ela deve ser avaliada de maneira particular em cada 
sujeito, pois cada um possui um contexto próprio de vida ao qual está inserido (Pereira, Alvarez \& Traebert, 2011).

Desenvolver a independência, a autonomia, o bem-estar psicológico e o sentimento de utilidade social é de suma importância para a melhora da qualidade de vida. Portanto, a manutenção da QV representa um fator fundamental para gerar um envelhecimento saudável. (Lima et.al, 2016; Brandão \& Zatt, 2015).

\section{Envelhecimento}

No contexto das relações sociais, observase mudanças relacionadas aos avanços do envelhecimento populacional e os impactos na estrutura social, que comprometem o envelhecimento ativo. O processo de envelhecer trouxe alterações em paradigmas sobre a saúde, o bem-estar, as classes comunitárias e o papel do indivíduo na sociedade (Spadoni- Pacheco \& Carvalho, 2019).

Didaticamente, pode-se dividir o envelhecimento em duas fases, o primário e o secundário. No primário, ocorre um processo gradual e inevitável de deterioração física que começa cedo na vida e continua ao longo dos anos; o secundário está relacionado a doenças,abusos e maus hábitos, fatores que em geral podem ser controlados (Papalia, \& Feldman, 2013). Outro conceito, amplamente utilizado, é o envelhecimento ativo da Organização Mundial da Saúde (OMS), que o define como a forma de otimização das oportunidades de saúde, socialização e segurança, tendo como foco a melhora da qualidade de vida no envelhecer (OMS, 2005).

O processo de envelhecimento do ser humano, está relacionado a três domínios gerais, são eles: o aumento dos déficits físicos; pressões e perdas sociais; e a perspectiva iminente de finitude. Entretanto, esse processo é heterogêneo e dependerá do estilo de vida adotado, considerando os aspectos históricos e culturais envolvidos, como também os históricos de comorbidades, fatores genéticos e ambientais (Beleza \& Soares, 2019).

Segundo Queiroz (2010), o processo de envelhecimento reflete diretamente em aspectos relacionados à qualidade de vida, como: capacidade funcional, o nível socioeconômico, o estado emocional, a interação social, a atividade intelectual, o autocuidado, o suporte familiar,o próprio estado de saúde, os valores culturais, éticos e a religiosidade, o estilo de vida, a satisfação com o emprego e/ou com atividades diárias e o ambiente em que se vive. Sendo que os idosos institucionalizados são mais vulneráveis a sofrerem perdas em relação a esses aspectos.

O idoso apresenta características inerentes a sua morfofisiologia, como maior prevalência a doenças crônicas e fragilidades, o que requer um cuidado especializado e estruturado para melhor adequação do indivíduo nessa fase da vida. Entretanto, a complexidadedo cuidar, associado aos novos arranjos familiares, levam muitas famílias a procurarem ILPI (Miranda, Mendes \& Silva, 2016). Com os novos arranjos familiares brasileiros e com o declínio da família nuclear ocasionados pelos efeitos socioeconômicos, demográficos, de saúde, tamanho da prole, separações, entrada da mulher no mercado de trabalho, celibato, mortalidade, viuvez, recasamentos e migrações, houve uma redução na perspectiva de envelhecimento em um ambiente familiar seguro. A mudança na constituição familiar aliada à entrada da mulher no mercado de trabalho fez com que ocorresse o desaparecimento do 
cuidador o que faz com quemuitas vezes o idoso more sozinho, com outros parentes ou em instituições de longa permanência para idosos. O grande número de idosos institucionalizados é consequência dessa diminuição do número de cuidadores (Queiroz, 2010).

\section{Instituições de Longa Permanência para Idoso}

Com a Constituição Federal de 1988, foi iniciado um processo de construção democrática das políticas públicas, destacando-se, entre elas, a Lei Orgânica da Assistência Social (Lei no 8742/73), a Política Nacional do Idoso (Lei no 8842/94) e o Estatuto do Idoso (Lei no 10.741/03) (Mendonça, 2006, p.183).

O Estatuto do Idoso, Lei n ${ }^{\circ} 10.741$ de $1^{\circ}$ de Outubro de 2003, Cap. II, Art. 49 prevê que:

As entidades que desenvolvam programas de institucionalização de longa permanênciaadotarão os seguintes princípios:

I- Preservação dos vínculos familiares;

II- Atendimento personalizado e em pequenos grupos;

III- Manutenção do idoso na mesma instituição, salvo em caso de força maior;

IV - Participação do idoso nas atividades comunitárias, de caráter interno e externo;

V- Observância dos direitos e garantias dos idosos;

VI- Preservação da identidade do idoso e oferecimento de ambiente de respeito e dignidade.
A defesa da cidadania, através de políticas públicas, tem a capacidade de gerar no indivíduo, e consequentemente na sociedade e Estado, o poder de união na construção de direitos sociais. Sendo assim, é dever dos representantes políticos, criarem formas de defesa da democracia e da cidadania e não priorizar interesses do capitalismo e grupos corporativos. Neste contexto, as políticas sociais devem ter como premissa os direitos dos cidadãos, em especial os vulneráveis, como as crianças, o índio, o negro e os idosos (Faleiros, 2005, citado por Mendonça, 2006).

Contudo, não existem políticas públicas definidas capazes de suprir a demanda de ILPI,deste modo a institucionalização por serviços filantrópicos ou privadas tornou-se comum (Souza, 2018). Instituições de Longa Permanência para Idosos (ILPIs) é uma denominação adotada pela Sociedade Brasileira de Geriatria e Gerontologia (SBGG) que surge para suprir otermo, abrigo, casa de repouso, asilo e similares. Atualmente, a procura por instituições asilaresou ILPI tornou-se crescente na sociedade. Esta demanda está associada a diversos fatores familiares e sociais complexos, e também relacionada as mudanças demográficas (Souza, 2018;Evangelista et al., 2014). Ressalta-se a importância e necessidade dessas políticas frente as novas estruturas familiares que estão em formação e ao crescimento da população de idosos (Souza, 2018).

Estudos realizados por Perlini, Leite e Furini (2007), Fonseca, Scoralick, Bertolino e Piazzolla (2011) encontraram como principais determinantes para a institucionalização de idosos o número reduzido de integrantes da família, ausência de condições físicas, financeiras e psicológicas para prestar o cuidado, o desejo do próprio idoso, síndrome demencial e problemas de 
relacionamento com os familiares, viuvez e múltiplas doenças.

Entretanto, é difícil a identificação, regulação e fiscalização dessas ILPI pelos órgãos governamentais, o que leva a insegurança de familiares que necessitam desses serviços e questionamento sobre a qualidade do serviço oferecido (Souza, 2018). Ao observar a estruturação histórica das ILPI, o seu alicerce traz um perfil assistencialista, em que os cuidados constituem apenas em ofertar alimentação e abrigo. Além disso, estrutura física inadequada juntamente com contratação de mão-de-obra desqualificada legalmente repercutem na assistência prestada e na QV desses idosos institucionalizados (Freitas \& Scheicher, 2010).

O processo de institucionalização do idoso é estressante e fator desencadeador de depressão (Freitas \& Scheicher, 2010). Além disso, deve-se considerar que idosos em espera por institucionalização podem apresentar mais fatores desencadeadores para desequilíbrios emocionais do que os já institucionalizados, devido ao fato de suas demandas de cuidados nãoserem supridas (Araújo \& Bós, 2017).

Dentro do campo teórico da gerontologia e das pesquisas voltadas ao conhecimento sobre o envelhecimento saudável, a depressão surge como uma das principais queixas dos

idosos (Hartmann Junior \& Gomes, 2014). Segundo Oliveira, Santos, Cruvinel e Néri (2006) adepressão tem sido considerada a doença psiquiátrica mais comum entre os idosos e a que mais afeta sua qualidade de vida.

Uma pesquisa realizada por Hartmann Junior, Gomes, Januário, Queiroz e Sougey (2012, citado por Hartmann Junior \& Gomes, 2014), intitulada "Qualidade de Vida e Depressãoem Idosas Institucionalizadas", foi visto que, dentre as idosas que participaram da pesquisa $15,8 \%$ não apresentaram sintomas característicos da depressão; $78,9 \%$ apresentaram uma pontuação que caracteriza depressão leve a moderada e 5,3\% depressão grave. O que segundo Hartmann Junior e Gomes (2014): Foi possível observar a prevalência dos sintomas avaliados e sua relação com os baixosíndices de qualidade de vida, como também a necessidade de intervenções voltadas parao contexto das ILPI e de ações direcionadas para a prevenção e a promoção da saúde neste contexto, visto que estas instituições nem sempre possuem serviço médico especializado para a prevenção e tratamento desta patologia (Hartmann Junior \& Gomes, 2014, p. 94).

Além disso, associado as alterações fisiológicas, psíquicas e socioeconômicas inerentes ao envelhecimento e, juntamente com as condições precárias das ILPI pode haver, de diversasmaneiras, o comprometimento da qualidade de vida de idosos institucionalizados. Deve-se considerar também, as rotinas rígidas e regras dessas instituições, que podem comprometer a autonomia do idoso e, consequentemente, a QV (Evangelista et al., 2014).

Sendo assim, este artigo tem por objetivo analisar à qualidade de vida de idosos em Instituições de Longa Permanência no Brasil.

\section{Método}

A revisão integrativa é uma metodologia que proporciona formas de sistematizar o conhecimento científico de determinado tema e demonstrar de forma linear a produção científica e a evolução do tema pesquisado. Além disso, fornece 
meios que possibilitem os leitores, avaliação e reprodutibilidade das técnicas utilizadas na elaboração da revisão (Botelho,Cunha \& Macedo, 2011).

Além do conhecimento do estado da arte do tema pesquisado, essa metodologiaoportuniza a incorporação de evidências na prática clínica (Mendes, Silveira \& Galvão, 2008). Uma das principais vantagens dessa metodologia é a possibilidade de inclusão de estudos

experimentais e não experimentais com diversos objetivos e teorias, tendo como consequência a observância de um mesmo tema por diferentes prismas (Gomes \& Caminha, 2014).

Esta revisão integrativa da literatura tem por finalidade analisar à qualidade de vida de idosos em instituições de longa permanência, nos últimos cinco anos, sendo desenvolvida em seis etapas: identificação do problema ou questionamento, estabelecimento de critérios de inclusão/exclusão de artigos, definição das informações a serem extraídas dos artigos selecionados, avaliação dos estudos incluídos na revisão integrativa, análise das informações, interpretação dos resultados, síntese do conhecimento ou apresentação da revisão (Botelho et al., 2011).

A pergunta norteadora para elaboração deste artigo foi: Como tem sido avaliada a qualidade de vida de idosos que vivem em Instituição de Longa Permanência?

A etapa coleta de dados ocorreu durante o mês de junho de 2019, utilizando-se a Biblioteca Virtual de Saúde (BVS), nas bases de dados: Literatura Latino-Americana e do Caribe em Ciências da Saúde (LILACS), Base de Dados em Enfermagem (BDENF), Bibliografia Brasileira de Odontologia (BBO), na base de dados Index Psi
Periódicos (IndexPsi)e demais bancos de dados que compõem a plataforma. Também foi realizado busca no ScientificEletronic Library Online de Saúde Pública (SCIELO). Os critérios inseridos foram: idioma português, publicados no período de 2014 - 2019.

Os Descritores em Ciências da Saúde (Decs) selecionados para a busca dos artigos foram: "Idoso", "Qualidade de vida", "Envelhecimento", "Saúde do Idoso Institucionalizado" e "Instituição de Longa Permanência para Idosos". Com intuito de reunir a maior quantidade de trabalhos realizouse duas buscas, a primeira com a associação dos Decs "Idoso", "Qualidadede vida", "Envelhecimento" e "Saúde do Idoso Institucionalizado" e a segunda com os Decs "Idoso", "Qualidade de vida", "Envelhecimento" e "Instituições de Longa Permanência para Idosos".

$\mathrm{Na}$ primeira busca encontrou-se um total de 26 artigos e na segunda 33, totalizando 59 artigos, conforme apresenta-se no fluxograma 1. Foram lidos os resumos e títulos para averiguação da consonância dos seguintes critérios de inclusão: artigos originais do tipo ensaios clínicos randomizados e não randomizados, estudos observacionais, estudos descritivos quantitativos e qualitativos, publicados nos anos de 2014 a 2019, no idioma português cujo focoe resultados estivessem de acordo com o objetivo do trabalho. Foram descartados artigos cujo o foco da qualidade de vida não estava relacionado ao idoso institucionalizado ou asilado, artigos de revisão bibliográfica, estudos de validação de instrumentos de qualidade de vida e que não contemplava o objetivo da pesquisa.

Ao final selecionou-se 6 artigos que foram lidos na íntegra e descritos em formato de quadro de acordo com autor e ano, base de dados, desenho do estudo, título, objetivo e amostra,instrumento de avaliação e principais resultados. 
Fluxograma 1. Percurso de busca da revisão

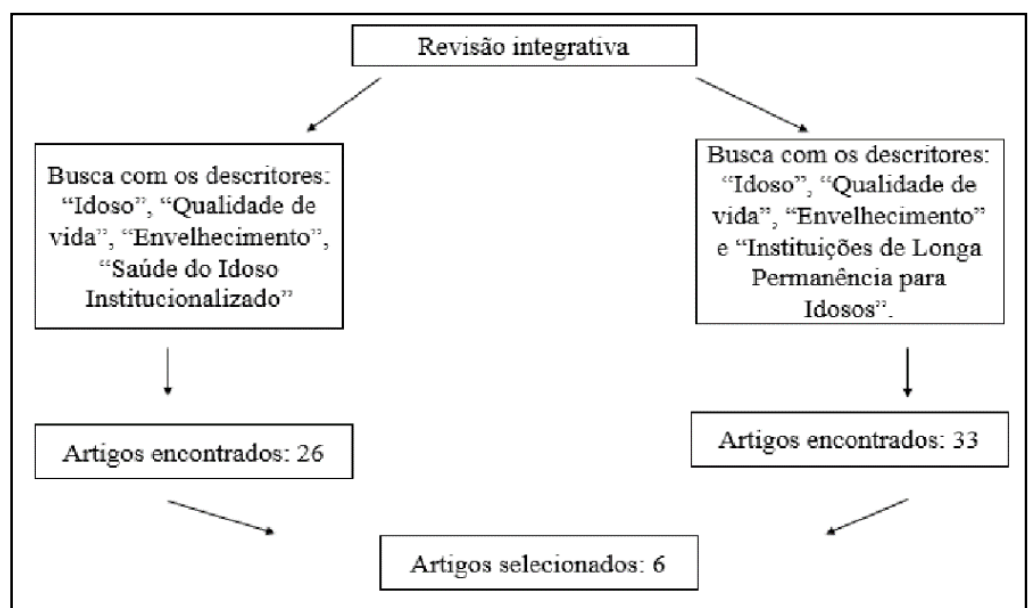

Fonte: Elaborado pelos autores

\section{Resultados e Discussões}

Os resultados encontrados e devidas localizações, conforme banco de dados, são apresentados na figura 1. Observa-se maior predomínio de trabalhos publicados na base de dados LILACS (29), seguida por IndexPsi (13), BDENF (9), SCIELO (4) e BBO (4). Contudo, apenas trabalhos das plataformas LILACS (3), IndexPsi (1) e BDENF (2) foram incluídos nessa revisão.

Em relação aos autores, haviam profissionais de diversas especialidades sendo $62 \%$ enfermeiros, $12 \%$ psicólogos, $8 \%$ nutricionistas, $8 \%$ médicos, $4 \%$ fisioterapeutas, $4 \%$ odontólogos e $4 \%$ estudantes de enfermagem.

No quadro 1 e 2 apresenta-se as principais características dos artigos incluídos nessa revisão. Dois deles foram publicados em 2016, dois em 2017, um em 2015 e um em 2018. Emrelação ao desenho do estudo, quatro possuíam abordagem quantitativa e dois qualitativos.

Figura 1. Distribuição dos artigos encontrados e selecionados por bases de dados.

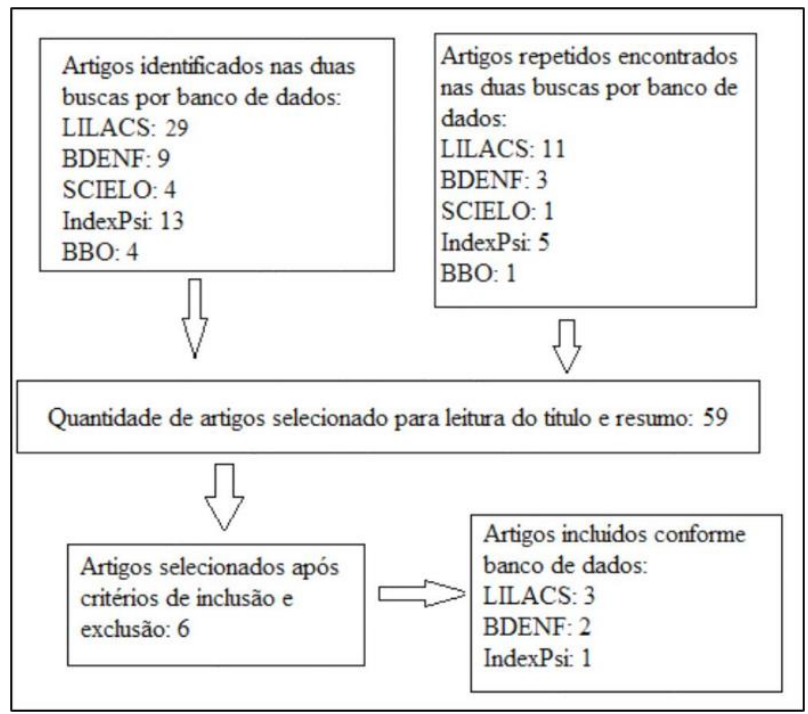

Fonte: Elaborado pelos autores 
Os de abordagem quantitativa utilizaram os questionários estruturados de avaliação da qualidade de vida o World Health Organization Quality of Life Assessment for Older Adults (WHOQOL-OLD) e o World Health Organization Quality of Life Bref (WHOQOL-BREF). Sendo que, O WHOQOLOLD é composto por 24 questões que contemplam seis domínios específicos para este grupo populacional: Funcionamento do Sensório, Autonomia, Atividades Passadas, Presentes e Futuras, Participação Social, Morte e Morrer, e Intimidade.

O instrumento WHOQOL-BREF é composto por 26 questões, sendo duas questões referentes à qualidade de vida geral e 24 questões divididas em quatro domínios que têm como objetivo analisar: a capacidade física, o bem-estar psicológico, as relações sociais e o meio ambiente no qual o indivíduo está inserido. Este instrumento apresenta mais um domínio, alémdos quatros, que analisa a qualidade de vida global.

Os trabalhos A1, A2, A3 e A6 tiveram uma abordagem quantitativa e utilizaram instrumentos validados para mensuração da qualidade de vida, o WHOQOL-OLD e WHOQOL-BREF, como citados acima. Estes permitem uma análise objetiva, quantificada e passível de comparação sobre qualidade de vida.

$\mathrm{O}$ artigo $\mathrm{A} 1$, foi o único a avaliar a $\mathrm{QV}$ de idosos asilados em instituições privadas de alto valor econômico, de acordo com autores a mensalidade custava em torno de 15 salários mínimos, e a instituição fornecia todo um aparato biopsicossocial, além de infraestrutura adequada a senilidade. Neste trabalho, a QV foi considerada satisfatória mesmo quando comparado a idosos não institucionalizados.

Pode-se associar a QV do idoso institucionalizado ao alto padrão econômico, em que idosos com maior poder aquisitivo apresentam melhores scores (Scherrer Júnior et al., 2018). Essa afirmação é corroborada pelos achados nos artigos A2, A3 e A6, em que os idosos institucionalizados tiveram piores resultados.

Quadro 1. Características dos artigos selecionados.

\begin{tabular}{|l|l|l|l|}
\hline Código / Autor -Ano & $\begin{array}{l}\text { Base de dados que os } \\
\text { artigos foram extraídos }\end{array}$ & Desenho doestudo & Título \\
\hline $\begin{array}{l}\text { A1 / Scherrer Júnioret al., } \\
2018\end{array}$ & BDENF & $\begin{array}{l}\text { Estudo quantitativo, } \\
\text { transversal }\end{array}$ & $\begin{array}{l}\text { Qualidade de vida de idosos residentes em } \\
\text { instituições privadas }\end{array}$ \\
\hline A2 / Araújo \& Bós,2017 & IndexPsi & $\begin{array}{l}\text { Estudo quantitativo } \\
\text { transversal }\end{array}$ & $\begin{array}{l}\text { Qualidade de vida da pessoa idosa conforme } \\
\text { nível de institucionalização }\end{array}$ \\
\hline A3 / Bassler et al.2017 & BDENF & $\begin{array}{l}\text { Estudo quantitativo } \\
\text { descritivo, transversal }\end{array}$ & $\begin{array}{l}\text { Avaliação da qualidade de vida de idosos } \\
\text { residentes em instituição de longa } \\
\text { permanênciapara idosos }\end{array}$ \\
\hline A4 / Lima et. al,2016 & LILACS & $\begin{array}{l}\text { Estudo descritivo, com } \\
\text { abordagemqualitativa }\end{array}$ & $\begin{array}{l}\text { Qualidade de vida sob a óptica dapessoa } \\
\text { idosa institucionalizada }\end{array}$ \\
\hline A5 / Brandão \&Zatt, 2015 & LILACS & $\begin{array}{l}\text { Estudo descritivo com } \\
\text { abordagemqualitativa }\end{array}$ & $\begin{array}{l}\text { Percepção de idosos, moradoresde uma } \\
\text { institução de longa permanência de um } \\
\text { município dointerior do Rio Grande do } \\
\text { Sul,sobre qualidade de vida }\end{array}$ \\
\hline A6 / Nogueira et al.,2016 & LILACS & $\begin{array}{l}\text { Estudo descritivo } \\
\text { quantitativo }\end{array}$ & $\begin{array}{l}\text { Comparando a qualidade de vidade idosos } \\
\text { institucionalizados e não institucionalizados }\end{array}$ \\
\hline
\end{tabular}

Fonte: Elaborado pelos autores 
Quadro 2. Principais resultados encontrados nos artigos selecionados para a revisão

\begin{tabular}{|c|c|c|}
\hline Código & Amostra / Objetivo & Instrumento de avaliação / Principais resultados \\
\hline A1 & $\begin{array}{l}\text { Participaram } 101 \text { idosos de quatro } \\
\text { instituições privadas. Objetivo: avaliar a } \\
\text { qualidade devida de idosos residentes em } \\
\text { instituições de longa permanência de alto } \\
\text { padrãoeconômico. }\end{array}$ & $\begin{array}{l}\text { Instrumento: WHOQOL-OLD / A percepção daqualidade de } \\
\text { vida demonstrou-se satisfatória, contudo, os piores resultados } \\
\text { foram no domínioda intimidade e o melhor é morte e morrer. }\end{array}$ \\
\hline $\mathrm{A} 2$ & $\begin{array}{l}\text { Analisado } 50 \text { idosos de cada grupo de } \\
\text { interesse. Objetivo: avaliar possíveis } \\
\text { diferenças na qualidade de vida da pessoa } \\
\text { idosa em lista de espera para residir em } \\
\text { ILPI, residente em ILPI, e daquele que não } \\
\text { está emlista de espera. }\end{array}$ & $\begin{array}{l}\text { Instrumentos: WHOQOL-BREF e WHOQOL- OLD / Idosos } \\
\text { institucionalizados não tiveram diferenças na piora da qualidade } \\
\text { de vida, entretanto a percepção da qualidade de vida já está } \\
\text { diminuída quando resolve-se procurar a institucionalização. }\end{array}$ \\
\hline A3 & $\begin{array}{l}\text { Pesquisado } 17 \text { idosos. Objetivo: avaliar a } \\
\text { qualidade de vida dos idosos residentes em } \\
\text { umaInstituição de LongaPermanência para } \\
\text { Idosos. }\end{array}$ & $\begin{array}{l}\text { Instrumentos: WHOQOL-BREF e o WHOQOL- } \\
\text { OLD / O estudo apresenta como regular a qualidade de vida } \\
\text { dos idosos institucionalizados. }\end{array}$ \\
\hline A4 & $\begin{array}{l}\text { Um total de } 8 \text { idosos participaram da } \\
\text { pesquisa. Objetivo: analisar o conceito } \\
\text { atribuído à qualidade de vidasob a ótica de } \\
\text { idosos institucionalizados. }\end{array}$ & $\begin{array}{l}\text { Questionário semiestruturado / Os autores apresentam vários } \\
\text { fatores que comprometem a qualidade de vida, como vida social, } \\
\text { cultura, saúde física e psicológica, humor e trabalho. }\end{array}$ \\
\hline A5 & $\begin{array}{l}\text { Amostra com } 8 \text { idosos. Objetivo: de } \\
\text { investigar a percepção sobre qualidade de } \\
\text { vida sob a perspectiva de idosos } \\
\text { institucionalizados. }\end{array}$ & $\begin{array}{l}\text { Questionário semiestruturado / Os autores observam o fato de } \\
\text { que as vivências e as relações sociais são fatores desencadeadores } \\
\text { deuma qualidade de vida, sendo que, alguns idososvalorizam suas } \\
\text { experiências vividas no processode institucionalização e o bom } \\
\text { relacionamento familiar. }\end{array}$ \\
\hline A6 & $\begin{array}{l}\text { Amostra: } 2 \text { grupos de idosos, sendo um } \\
\text { institucionalizado e o outro não, com } 20 \\
\text { participantes em cada. Objetivo: avaliar } \\
\text { comparativamente a qualidade de vida de } \\
\text { idosos institucionalizados e não } \\
\text { institucionalizados. }\end{array}$ & $\begin{array}{l}\text { Instrumento WHOQOL-OLD / Neste trabalho os idosos } \\
\text { institucionalizados tiveram piores desempenhos nos domínios } \\
\text { de funcionamento sensório; autonomia; atividades passadas, } \\
\text { presentes e futuras; participação social; e intimidades. }\end{array}$ \\
\hline
\end{tabular}

Fonte: Elaborado pelos autores

Em relação ao A2, que avaliou a qualidade de vida de idosos institucionalizados, emlista de espera para institucionalização e não institucionalizados, os idosos em espera tiveramos piores scores na avaliação, quando comparado aos institucionalizados e os não institucionalizados, tendo este último os melhores scores totais. Este foi o único trabalho queavaliou a qualidade de vida de idosos em lista de espera para entrar em ILPI. Entende-se que o idoso que necessita de institucionalização e não consegue esses serviço encontra-se em piores condições do que os institucionalizados, tal achado demostra a necessidade de mais estudos e análise dessa população e a importância do acesso aos serviços asilares (Araújo \& Bós, 2017).Os artigos A3 e A6, também apresentam scores mais baixos para idosos que residem

ILPI, do que os que não residem. Residir em uma instituição asilar requer do idoso rotinas pré estabelecidas, regras, perda da autonomia, stresse, o que pode gerar isolamento social e que quando associados podem ter como consequência a piora da QV (Evangelista et al., 2014). Porém, vale ressaltar os achados no artigo A1, em que mesmo institucionalizados, idosos com alto padrão econômico tiveram níveis de QV considerados pelos autores como satisfatório (Scherrer Júnior et al., 2018).

Os trabalhos com abordagem qualitativa, 
A4 e A5, utilizaram questionário semiestruturados, com técnica de Análise de Conteúdo. Esses artigos referem-se a um estudo sobre a percepção dos idosos que residem em instituição de longa permanência sobre a qualidade de vida.

Do artigo A4, emergiu três categorias de análise: percepção do idoso sobre QV; comportamentos que favorecem a QV; e a concepção do idoso sobre o envelhecimento saudável. Através dessas perspectivas, entende-se que a independência, a autonomia, o bem- estar psicológico e o sentimento de utilidade social tem forte ligação com as dimensões da QV(Lima et al., 2016).

Em relação ao artigo $\mathrm{A5}$, dividiu-se a entrevista em categorias, dentre elas questões relacionadas a qualidade de vida, relações sociais, atividade física, espiritualidade, suporte familiar, habilidade funcional e qualidade de vida na instituição. Este estudo corrobora com osachados do A4 em que o fato de manter boas relações sociais contribui para uma melhor QV (Lima et al., 2016; Brandão \& Zatt, 2015).

A percepção sobre qualidade de vida pode ser influenciada por diversos fatores que envolvem dimensões emocionais, sociais, culturais e físicas. Entende-se que, sob o prisma dosidosos a qualidade de vida está relacionada com o fato de poder viver bem, se sentir bem para aproveitar o que a vida lhes oferece e buscar manter relacionamentos sociais (Brandão \& Zatt,2015).

Segundo Brandão e Zatt (2015), envelhecer com qualidade de vida é uma preocupaçãopara os profissionais da área da saúde. Vê-se a necessidade de aprofundar os estudos com relação à qualidade de vida na terceira idade, para assim avaliar o que eles consideram importante para viverem felizes e satisfeitos nesse período, onde todos merecem viver e conviver em um ambiente onde possam vivenciar suas expectativas, realizar seus sonhos, para que consigam viver com qualidade.

A partir das análises dos resultados, desconsiderando os idosos com alto padrão econômico, compreende-se que a qualidade de vida dos idosos institucionalizados é inferior aos dos idosos não institucionalizados.

\section{Considerações Finais}

$\mathrm{O}$ aumento da expectativa de vida associada as novas estruturas familiares em formação, gera uma carência de arranjos sociais que consigam atender a demanda de idosos que passam a necessitar de Instituição de Longa Permanência para Idosos.

Nesta fase da vida, é necessário um suporte de cuidados que supra as necessidades de vida diária, como alimentação, higiene e aporte emocional e social para que o idoso consiga uma qualidade de vida satisfatória. Aos que não estam inseridos em ambientes familiares, as ILPI deveriam ser locais adequados para esses cuidados.

Entretanto, essas instituições não são capazes de atender os idosos em sua ampla carência de cuidados biopsicossocial, visto que, os idosos asilados tiveram qualidade de vida menor quando comparado a outros não institucionalizados. Porém, o poder aquisitivo econômico foi um fator protetor para qualidade de vida, sendo que, idosos em instituições de alto padrão econômico tiveram a qualidade de vida similares a idosos não asilados.

Em função do constado e refletido neste escrito, entende-se que faz-se mister a ampliação dos estudos relacionados a vida senil, no intuito de influenciar e quiçá mobilizar o poder público, em especial, na implementação de medidas que melhorem a qualidade de vida dos idosos, como por exemplo, por 
meio de ILPI que ofereça serviços de excelência.

\section{Referências}

A. G. S. (2018). Qualidade de vida de idosos residentes em instituições privada. 12(8), 2113-2119. doi: 10.5205/1981-8963-v12i8a234536p2113-21192018

A. P. (2018). The life hope of elderly: profile assessment and Herth Scale. Revista de pesquisa: cuidado é fundamental [online], 1, 167-172. doi: 10.9789/2175- 5361.2018.v10i1.167-172

Araújo, A. M., \& Bós, Â. J. G. (2017). Qualidade de vida da pessoa idosa conforme nível de institucionalização. Estudos interdisciplinares sobre o envelhecimento, 22(3), 137-152. Retrieved from https://seer.ufrgs.br/RevEnvelhecer/article/vi ew/60224/49694

Baltes, P.; Lindenberger, U.; Stadinger, U. (2006). Lifespan theory in developmental psychology. In R. LERNER. (Ed.) Handbook of Child Psychology. Vol.1: Theoretical Models of Human Development. Hoboken, NJ: Wiley. 569-664.

Bassler, T. C., Santos, F. R., Junior, A. G. S., Furlan, M. C. R., \& Maia, C. R. (2017). Avaliaçãoda qualidade de vida de idosos residentes em instituição de longa permanência para idosos. Revista enfermagem UFPE [online], 11(1), 10-17. doi: 10.5205/reuol.9978- 88449-6-1101201702

Beleza, C. M. F., \& Soares, S. M. (2019). A concepção de envelhecimento com base na teoria de campo de Kurt Lewin e a dinâmica de grupos. Ciência es Saúde Coletiva, 24(8), 3141- 3146. doi: 10.1590/1413-812332018248.30192017

Bimler, D., \& Uusküla, M. (2018). Individual variations in color-concept space replicate acrosslanguages. Journal of the Optical Society of America A, 35(4), 184. Doi:10.1364/josaa.35.00b184

Botelho, L. L. R., Cunha, C. C. de A., \& Macedo, M. (2011). O Método da revisão integrativa nos estudos organizacionais. Gestão e Sociedade, 5(11), 121. doi: $10.21171 /$ ges.v5i11.1220

Brandão, V. C., \& Zatt, G. B. (2015). Percepção de idosos, moradores de uma instituição delonga permanência de um município do interior do Rio Grande do Sul, sobre qualidade de

$\begin{array}{ll}\text { vida. } & \text { Aletheia, } \\ \text { 46, } & 90-102 . \\ \text { Retrieved } & \text { from }\end{array}$

http://pepsic.bvsalud.org/scielo.php?script $=$ sci _arttext\&pid=S1413-03942015000100008

Brasília: Organização Pan-Americana de Saúde (OPAS); 2005.

Campolina, A. G., Adami, F., Santos, J. L. F., \& Lebrão, M. L. (2013). A Transição de saúde eas mudanças na expectativa de vida saudável da população idosa: Possíveis impactos da prevenção de doenças crônicas. Cadernos de Saúde Pública, 29(6), 1217-1229. doi: 10.1590/S0102311X2013000600018

Celich, K.L.S. (2008). Domínios de qualidade de vida e capacidade para a tomada de decisão em idosos participantes de grupos da terceira idade (Tese de Doutorado). Retrieved from http://repositorio.pucrs.br/dspace/handle/109 $23 / 3587$

Evangelista, R. A., Bueno, A. de A., Castro, P. A., Nascimento, J. N., Araújo, N. T., \& Aires,

Fonseca, A. C. C., Scoralick, F. M., Silva, C. L. D., Bertolino, A. C., Palma, D. P., \& Piazolla,

Freire, H. S. S. (2018). Conhecimento dos cuidadores de idosos institucionalizados sobre prevenção de quedas em Fortaleza - CE. Revista Nursing, 21(242), 2248-2253. Retrieved from https://pesquisa.bvsalud.org/portal/resource/ pt/biblio-911167

Freitas, M. A. V. \& Scheicher, M. E. (2010). Qualidade de vida de idosos institucionalizados. Revista Brasileira de Geriatria e Gerontologia, 13(3), 395-401. doi: 10.1590/s1809- 98232010000300006

G. P. (2014). Perceptions and experiences of elderly residents in a nursing home. Revistada escola de enfermagem da USP, 48(2), 81-86. doi:10.1590/s0080- 623420140000800013

Gomes, I. S. \& Caminha, I. O. (2014). Guia para estudos de revisão sistemática: Uma opção metodológica para as ciências do movimento humano. Revista de educação física da UFRGS, 20 (01), 395-411. doi: 10.22456/1982-8918.41542

Hartmann Junior, J. A. S.; Gomes, G. C. (2014). Depressão em idosos institucionalizados: as 
singularidades de um sofrimento visto em sua diversidade. Revista SBPH 17(2). Retrieved from http://pepsic.bvsalud.org/scielo.php?script=sci _arttext\&pid=S1516-08582014000200006

Instituto Brasileiro De Geografia E Estatística - IBGE. (2011). Sinopse do Senso Demográficode 2010.

L. P. (2011). Perfil epidemiológico de idosos e fatores determinantes para a admissão eminstituições de longa permanência no Distrito Federal. Brasília Med,48(4), 366-371. Retrieved from http://www.rbm.org.br/details/238/pt-

$\mathrm{BR} /$ perfil-epidemiologico-de- idosos-e-fatoresdeterminantes-para-a-admissao-em-instituicoesde-longa-permanencia-no-distrito-federal

Lei $\mathrm{n}^{\circ} 10.741$ de $1^{\circ}$ de outubro de 2003. (2003, 1 outubro). Dispõe sobre o Estatuto do Idoso edá outras providências. Diário Oficial da União, Brasília.

Lima, A. P. M., Gomes, K. V. L., Frota, N. M., \& Pereira, F. G. F. (2016). Qualidade de vida sob a óptica da pessoa idosa institucionalizada. Revista Brasileira em promoção da saúde, 29(1), 14-19. Retrieved from http://periodicos.unifor.br/RBPS/article/view /4239/pdf

Matos, M. A. B., Alves Barbosa, B. L., Costa, M. C., Rocha, F. C. V., Landim Almeida, C. A.P., \& Amorim, F. C. M. (2019). As Repercussões causadas pela incontinência urinária naqualidade de vida do idoso. Revista de pesquisa: cuidado é fundamental [online], 11(3), 567. doi:10.9789/2175-5361.2019.v11i3.567-575

Mendes, K. Da S., Silveira, R. C. C. P., \& Galvão, C. M. (2008). Revisão Integrativa: Método de pesquisa para a incorporação de evidências na saúde e na enfermagem. Texto \& Contexto - Enfermagem, 17(4), 758-764. doi:10.1590/S010407072008000400018

Mendonça, J. (2006). Os Conselhos e o Protagonismo do Idoso Como Exercício Ativo da Cidadania. In. Cortê B., Mercadante E. F. \& Arcuri I. G. (Orgs), Envelhecimento e velhice: um guia para a vida (pp. 183-196). São Paulo: Vetor.

Miranda, G. M. D., Mendes, A. C. G., \& Silva, A. L. A. (2016). Population aging in Brazil: current and future social challenges and consequences. Revista Brasileira de Geriatria e Gerontologia, 19(3), 507-519. doi:10.1590/1809-
98232016019.150140

Nogueira, M. F., Maria, I., \& Torquato, B. (2016). A Dança como contributo para a qualidade de vida de idosos institucionalizados e não institucionalizados. Revista da unidade de investigação instituto politécnico de Santarém, 4(2), 254-272. doi: 10.25746/ruiips.v4.i2.9936

Oliveira, K. L., Santos, A. A. A., Cruvinel, M., \& Néri, A. L. (2006). Relação entre ansiedade, depressão e desesperança entre grupos de idosos. doi: 10.1590/S1413- 73722006000200014

Oliveira, L. de M., Silva, S. M. S., Lima, E. de F. A., Gomes, M. das G. C., \& Olympio, P. C.

OMS (1998). Promoción de la salud. Glosario. Journal of Chemical Information and Modeling, 53(9), 16891699. doi: $10.1017 / \mathrm{CBO} 9781107415324.004$

Organização Mundial da Saúde (OMS). Envelhecimento Ativo: Uma política de saúde.

Papalia, D. E, Feldman, R. D. (2013). Desenvolvimento Humano (p.573) 12. Edição Porto Alegre: AMGH, 2013.

Pavan, F. J., Meneguel, S. N., \& Junges, J. R. (2008). Mulheres Idosas Enfrentando a Institucionalização. Cadernos de Saúde Pública, 24(9). doi: 10.1590/S0102- 311X2008000900025

Pereira, K. C. R., Alvarez, A. M., \& Traebert, J. L. (2011). Contribuição das condições sociodemográficas para a percepção da qualidade de vida em idosos. Revista Brasileira de Geriatria e Gerontologia, 14(1), 85-95. doi: 10.1590/s180998232011000100010

Perlini, N. M. O. G., Leite, M. T., \& Furini, A. C. (2007). Em busca de uma instituição para a pessoa idosa morar: motivos apontados por familiares. Revista da Escola de Enfermagem, 41(2), 229-236. doi:10.1590/S0080-62342007000200008

Queiroz, G. A. (2010). Qualidade de vida em instituições de longa permanência para idosos: considerações a partir de um modelo alternativo de assistência (Dissertação de Mestrado). Retrieved from br/portal2 https://ufsj.edu. 
repositorio/File/mestradopsicologia/2010/Dis sertacoes/Dissertacao_Gleicimara\%20.pf

Scherrer Júnior, G., Okun, M. F. P., Passos, K. G., Ernandes, R. C., Alonso, A. C., \& Belasco,

Schiozer, W. (2011). Tratamento de queimaduras em pacientes idosos. Revista Brasileira de Queimaduras. 10(4),

105.

Retrieved

from

http://www.rbqueimaduras.com.br/details/77/ $\mathrm{pt}-\mathrm{BR} /$ tratamento-de-queimaduras-empacientes-idosos

Schneider, R. H., \& Irigaray, T. Q. (2008). O Envelhecimento na atualidade: aspectos cronológicos, biológicos, psicológicos e sociais Retrieved from http://www.scielo.br/scielo.php?script=sci_art text\&pid=S0103166X2008000400013\&lng=en\&nrm=iso\&tlng $=\mathrm{pt}$

Scoralick-Lempke, N. N., \& Barbosa, A. J. G. (2012). Educação e envelhecimento: contribuições da perspectiva Life-Span. Estudos de Psicologia (Campinas), 29(1), 647- 655. doi:10.1590/s0103166x2012000500001

Souza, M. C. M. R., Horta, N. C., Cunha, M. C. M., Ferreira, Q. N., Barral, T. T. L., \& Oliveira,

Spadoni-Pacheco, L. M., \& Carvalho, G. A. (2019). Quality of life and self-esteem in elderly patients who did or did not have cosmetic surgery. Revista Brasileira de Cirurgia Plástica (RBCP) - Brazilian Journal of Plastic Sugery, 33(4), 528-535. doi:10.5935/2177- 1235.2018rbcp0174

T. R. P. R. (2018). Instituições de longa permanência para idosos: A Distribuição espacial na região metropolitana de Belo Horizonte. Geriatrics, Gerontology and Aging, 12(2), 68-73. doi: $10.5327 / \mathrm{z} 2447-211520181800019$

Torres, G. V., Reis, L. A., Reis, L. A. \& Fernandes, M. H. (2009). Qualidade de vida e fatoresassociados em idosos dependentes em uma cidade do interior do nordeste. Jornal brasileiro de psiquiatria 58(1). doi: 10.1590/S0047-20852009000100006

United Nations. (2011). World population ageing: 19502050. 